\title{
Sentinel Lymph Node Biopsy for Melanoma: A Plea to Let the Data Speak
}

\author{
Daniel Coit, MD, FACS \\ Memorial Sloan-Kettering Cancer Center, New York, NY
}

Twenty-two years ago, Morton and colleagues reintroduced the world to a deceptively simple concept: that the initial regional node to which a melanoma would metastasize (if it was going to do so) could reliably be identified, removed, and examined to provide important prognostic information and help guide treatment decisions for patients with this diagnosis. ${ }^{1}$ At a time when routine elective lymph node dissection had been largely abandoned as excessively morbid with no impact on survival, the robust and reproducible technique of sentinel lymph node biopsy (SLNB) was rapidly adopted in the initial management of patients presenting with localized invasive melanoma; further, clinical relevance was soon found in guiding the treatment of many more patients with breast cancer.

In the interim, while others have either analyzed retrospective series or criticized the procedure, no other single physician devoted more time and energy than Dr. Morton to rigorously and critically evaluate the role of SLNB in melanoma, conceiving and completing two seminal international prospective randomized surgical trials, each accruing nearly 2,000 patients, to define the value of initial management with SLNB (MSLT-1) and, among patients with a positive SLNB finding, the role of completion lymph node dissection (MSLT-2). The final report of findings from MSLT-1, published in the New England Journal of Medicine, has provided clinicians with invaluable, highquality information to interpret and use in the management of their melanoma patients. ${ }^{2}$ I write to express both my enormous appreciation for the opportunity to examine

(C) Society of Surgical Oncology 2014

First Received: 17 March 2014;

Published Online: 25 July 2014

D. Coit, MD, FACS

e-mail: coitd@mskcc.org these data—but also my concern about how the authors have presented this information.

The final report of MSLT-1 has largely confirmed the principal findings of the initial report published in 2006 on patients with intermediate-thickness melanomas (1.2$3.5 \mathrm{~mm}$ ) and provides new information on patients with thick melanoma $(>3.5 \mathrm{~mm}){ }^{3}$ Initial management with wide excision (WE) and SLNB is not associated with any improvement in melanoma-specific survival, the primary end point of the trial, compared to initial management with WE and nodal basin observation (OBS). This is a negative trial. This result should not surprise us, because the trial design of MSLT-1 was essentially identical to that of the three other major randomized trials of elective lymph node dissection with negative findings, with the principal difference being that the patients in MSLT-1 with negative sentinel nodes at presentation were spared the morbidity of elective lymphadenectomy. ${ }^{4-6}$

The current report confirmed an improvement in recurrence-free survival after SLNB, a secondary end point of the trial, for patients with both intermediate-thickness and thick melanomas. As clearly shown in the article's supplementary appendix, this difference was primarily due to an expected increased incidence of initial nodal failure in the OBS group. Although this is listed as one of the end points that "validate" the use of SLNB, the authors fail to provide information on the ultimate clinical relevance of improved nodal basin control after SLNB. The authors might cite the potential for incrementally higher complication rates after therapeutic LND for a clinically positive node compared to completion LND for a positive sentinel node, and an incrementally higher rate of nodal basin relapse after therapeutic LND than after completion LND. However, these events will affect only a small subset of the subset of patients with positive nodes. How many more actual patients in the OBS group experienced more complications following therapeutic LND, or ultimate loss of 
control in the nodal basin, compared to the SLNB patients? Did this exceed the small but finite number of patients with complications seen among all those who underwent SLNB? Is that sufficient impetus for all patients to undergo SLNB?

Much has been written criticizing the preplanned subset analysis of the outcome of patients who ultimately developed nodal metastases. Using the statistical gymnastics of "accelerated failure time latent subgroup analysis" (how many of the few who will actually look up these references will sufficiently understand the sophisticated statistical methodology to appreciate its clinical relevance?), this report has confirmed the previous finding that among node positive patients with intermediate-thickness melanoma, disease-specific survival is better when the nodal metastases are found at SLNB. ${ }^{7,8}$ Not reported previously, and conspicuously omitted from this report's conclusion, is the fact that a similar improvement in survival was not observed among node-positive patients with thick melanoma. This is new and useful information that easily could have been highlighted in the abstract and/or conclusion of the report.

We need to be cautious about how we interpret these data for patients with intermediate-thickness melanoma. In their conclusion, the authors refer to a "substantial" improvement in survival among node-positive patients when the metastases are found at SLNB. Even if we accept the hypothesis (and many will not) that all patients with a positive SLN are destined to develop clinically positive nodes, the potential impact of this OBS on the group of all patients with intermediate-thickness melanoma who are confronting the decision to undergo SLNB is unclear. For these patients, the "substantial" 15-20\% improvement in survival will apply to only the $20 \%$ of patients with positive SLN's. This results in a potential benefit for, at most, 3-4\% of the initial population. The magnitude of this potential benefit has appeared with remarkable consistency in retrospective subset analysis of node-positive patients across all prospective randomized controlled trials evaluating the impact of early nodal intervention in patients with intermediate-thickness melanoma. ${ }^{4-6}$ If we accept the consistency of this calculation-and particularly as we increasingly accept the heterogeneous genetics, pathogenesis, and biologic potential of individual melanomas-then there may indeed be a small proportion of patients, not yet identifiable at presentation, in whom early intervention in the regional nodal basin might interrupt a pathway of melanoma progression, a metastatic cascade, that could result in improved disease-specific survival. Rather than term the impact on survival "substantial" when we speak with our patients, we would be more transparent to acknowledge that at best, SLNB could impact the outcome of a small minority of patients with intermediate-thickness melanomas-a minority so small as to be undetectable in this trial.
The study highlights the fact that although the procedure of SLNB may be quite accurate, it misses $20 \%$ of positive nodes in patients with intermediate-thickness melanoma and $17 \%$ of positive nodes in patients with thick melanomas. Furthermore, the report again highlights the poor prognosis of these patients with a negative SLNB who go on to subsequent clinical nodal basin failure. As expected, when these patients with falsely negative disease are included with the patients with truly negative findings in an intention-to-treat analysis, the magnitude of the "substantial benefit" associated with SLNB was shown to be less significant. Perhaps we should be sharing this information with our patients at the time we discuss and offer SLNB.

The report reaffirms that SLNB is an effective staging procedure, and one that currently provides more important prognostic information than can be derived from characteristics of the primary tumor. Curiously, while the article presents the multivariate hazard ratios confirming the importance of SLNB status as the strongest independent predictor of recurrence-free and melanoma-specific survival among patients with intermediate-thickness melanoma, a similar table detailing the prognostic significance of the SLN status among patients with thick melanoma is not presented, either in the body of the article or in the supplemental appendix.

The prognostic information derived from SLNB will assume increasing clinical importance if we can ever develop an effective adjuvant treatment to address the increased risk for patients with a positive SLN. In contrast, that prognostic information will be less important if we can derive more reliable, reproducible, accurate, and possibly genetic predictors of outcome from characteristics of the primary tumors. Only the future will tell.

There is an assumption that the finding of melanoma cells in the sentinel node is always prognostic and never a clinical false-positive finding. That assumption is generally supported by data shown in their Fig. 3b, where $42 \%$ of patients with thick melanoma will ultimately be found to have a positive regional node, either after SLNB and follow-up, or after initial OBS alone. However, close examination of their Fig. 3a may support a slightly different hypothesis. Among patients with intermediatethickness melanoma, the ultimate incidence of a "positive" node is $2.4 \%$ higher after SLNB and follow-up compared to OBS alone. Although some might argue that all of the nodal failures have not yet been seen in this lower-risk, later-recurrence-prone population, the two curves in this figure are remarkably parallel when followed out beyond 5 or 6 years. A credible alternate hypothesis might be that SLNB identified up to $2.4 \%$ of patients who were not destined to develop clinical nodal metastases. Compared to patients with thick melanoma whose positive sentinel 
nodes usually contain significant tumor burden, patients with intermediate-thickness melanoma may have positive sentinel nodes with only isolated tumor cells; some might even have had nodal nevi or histiocytes that were overinterpreted as melanoma cells. As with any staging test, we must acknowledge the potential for the phenomenon of a false-positive result, even if it is infrequent, especially if it leads to significant interventions such as completion lymph node dissection and adjuvant systemic therapy.

I conclude this editorial with disclosures. First, we continue to discuss and offer SLNB to accurately stage our patients with melanoma; we do not represent any survival advantage associated with this procedure. Second, Morton was a true giant in his field, a leader who contributed much to many. He was a patient teacher and a physician completely devoted to improving the well-being of his patients. He was a tireless translational researcher long before the term was fashionable. He brought countless new ideas and laboratory findings into the clinic, always for the potential benefit of his patients, but he never accepted any as effective without rigorous clinical evaluation. Further, he was my mentor, my colleague, and my good friend. Whenever we disagreed on clinical or academic issues, we always did so openly and with mutual respect. Now, as always, we owe Dr. Morton and his memory the respect of our honest appraisal of the extraordinary facts his trial has provided. We need to let these critically important data speak to us freely so that we can draw reasonable and rational conclusions about the role and the limitations of SLNB in the initial management of our patients with melanoma.

\section{REFERENCES}

1. Morton DL, Wen DR, Wong JH, et al. Technical details of intraoperative lymphatic mapping for early stage melanoma. Arch Surg. 1992;127:392-9.

2. Morton DL, Thompson JF, Cochran AJ, et al. Final trial report of sentinel-node biopsy versus nodal observation in melanoma. $N$ Engl J Med. 2014;370:599-609.

3. Morton DL, Thompson JF, Cochran AJ, et al. Sentinel-node biopsy or nodal observation in melanoma. N Engl J Med. 2006;355:130717.

4. Veronesi U, Adamus J, Bandiera DC, et al. Inefficacy of immediate node dissection in stage 1 melanoma of the limbs. $N$ Engl J Med. 1977;297:627-30.

5. Cascinelli N, Morabito A, Santinami M, et al. Immediate or delayed dissection of regional nodes in patients with melanoma of the trunk: a randomized trial. Lancet. 1998;351:793-6.

6. Balch CM, Soong S, Ross MI, et al. Long term results of a multiinstitutional randomized trial comparing prognostic factors and surgical results for intermediate thickness melanomas (1.0 to $4.0 \mathrm{~mm})$. Ann Surg Oncol. 2000;7:87-97.

7. Altstein L, Li G, Elashoff RM. A method to estimate treatment efficacy among latent subgroups of a randomized clinical trial. Stat Med. 2011;30:709-17.

8. Altstein L, Li G. Latent subgroup analysis of a randomized clinical through a semiparametric accelerated failure time mixture model. Biometrics. 2013;69:52-61. 